\title{
Article \\ Three-Dimensional Broadband and Isotropic Double-Mesh Twin-Wire Media for Meta-Lenses
}

\author{
Hairu Wang (D), Qiao Chen *D, Oskar Zetterstrom (D) and Oscar Quevedo-Teruel (D) \\ Division of Electromagnetic Engineering, KTH Royal Institute of Technology, 11428 Stockholm, Sweden; \\ hairu@kth.se (H.W.); oskarz@kth.se (O.Z.); oscarqt@kth.se (O.Q.-T.) \\ * Correspondence: qiaoc@kth.se
}

Citation: Wang, H.; Chen, Q.;

Zetterstrom, O.; Quevedo-Teruel, O

Three-Dimensional Broadband and Isotropic Double-Mesh Twin-Wire Media for Meta-Lenses. Appl. Sci. 2021, 11, 7153. https://doi.org/ 10.3390/app11157153

Academic Editor: Shah Nawaz Burokur

Received: 30 June 2021

Accepted: 29 July 2021

Published: 3 August 2021

Publisher's Note: MDPI stays neutral with regard to jurisdictional claims in published maps and institutional affiliations.

\begin{abstract}
Lenses are used for multiple applications, including communications, surveillance and security, and medical instruments. In homogeneous lenses, the contour is used to control the electromagnetic propagation. Differently, graded-index lenses make use of inhomogeneous materials, which is an extra degree of freedom. This extra degree of freedom enables the design of devices with a high performance. For instance, rotationally symmetric lenses without spherical aberrations, e.g., the Luneburg lens, can be designed. However, the manufacturing of such lenses is more complex. One possible approach to implement these lenses is using metamaterials, which are able to produce equivalent refractive indices. Here, we propose a new type of three-dimensional metamaterial formed with two independent sets of wires. The double-mesh twin-wire structure permits the propagation of a first mode without cut-off frequency and with low dispersion and high isotropy. These properties are similar to periodic structures with higher symmetries, such as glide symmetry. The variations of the equivalent refractive index are achieved with the dimension of the meandered wires. The potential of this new metamaterial is demonstrated with simulated results of a Luneburg meta-lens.
\end{abstract}

Keywords: glide symmetry; meta-lens; double-mesh; wire medium; broadband response; isotropic response; metamaterials; Luneburg lens

\section{Introduction}

Many emerging technologies, e.g., high-resolution radars [1], and the next generation of mobile communications [2], are expected to operate at higher operational frequencies (above $15 \mathrm{GHz}$ ). At these frequencies, the propagation loss is high. To mitigate this loss, high gain antennas must be used. In some of these applications, an angular coverage is also requested; therefore, these high-gain antennas require beam steering where low scan losses are an asset.

A directive beam can be produced with a lens antenna. This type of antenna employs a lens to shape the radiation from a feeding element. Typically, the feeding element is placed at the focal point/line of the lens, and the lens transforms the radiation from the feed into a planar wave. A wide angular beam coverage with low scan losses can be obtained using rotationally symmetric lenses with multiple feeds placed along the focal line [3]. Due to the simple feed system, lens antennas can be cost-effective at high frequency [4,5]. For example, in [1], a homogeneous spherical lens antenna with low scan losses in a $180^{\circ}$ range is proposed. Similarly, in [6], a wide scanning homogeneous cylindrical lens antenna is designed. While a wide scan range is obtained in these homogeneous lenses, they suffer from spherical aberrations leading to low aperture efficiency and high side lobes. To overcome these limitations, a graded index lens can be used.

In a graded index lens, the refractive index varies as a function of space. Traditionally, graded index lenses were difficult to manufacture. Three-dimensional (3D) graded index lenses were manufactured in concentric hemispherical layers, which is inconvenient and costly $[7,8]$. Recently, it has been demonstrated that 3D graded index lenses can be manufactured using additive manufacturing (i.e., 3D printing) by spatially varying the density 
of the deposited dielectric material [9-14]. However, the use of dielectric introduces losses that are prohibitive at high frequency. A mostly metallic 3D-graded index lens is designed in [15]. However, the designed lens exhibits strong anisotropy, which degrades the scanning performance of the lens. Alternatively, two-dimensional (2D)-graded index lenses can be realized using concentric dielectric cylinders $[16,17]$ or mimicked with quasi-periodic structures [18-23] or geodesic surfaces [5,24-26]. However, the scanning is reduced to one dimension in the 2D lenses. Furthermore, the aperture of the 2D lenses is limited in one direction, which makes it difficult to reach gain values above $30 \mathrm{dBi}$.

Although fully-metallic 3D printers are still expensive, researchers are actively working to make this technology cost effective $[27,28]$. A cheaper solution is to produce fully metallic structures with metallic coating [29-31]. With these techniques, fully metallic complex 3D structures can be manufactured, which allows for the design of cost-effective metallic 3D-graded index lenses. The wire mesh is one such complex structure that has received attention by researchers due to its exotic electromagnetic behavior [32-34]. A single-wire mesh structure has all its metallic wires connected, which results in the structure having a cut-off frequency. The propagating modes in this structure are highly dispersive, and it is therefore not suitable for lens design. On the other hand, a double-wire mesh structure has two independent wire meshes and can support wave propagation without cut-off frequency with moderate dispersion [34].

In this paper, we investigate the double-mesh twin-wire structure. We demonstrate that the dispersion can be reduced by off-shifting the two wire meshes with half the period in three Cartesian directions, similar to what has been observed in glide-symmetric structures [35-37]. We apply this double-mesh twin-wire structure in the design of a fully metallic graded index Luneburg lens.

\section{Dispersion Analysis of the Double-Mesh Twin-Wire Structure}

To aid in the fundamental understanding of the structure behaviors, we demonstrate the evolution of the proposed double-mesh twin-wire unit cell in Figure 1 and study the corresponding dispersion characteristics of those evolutionary geometries by examining their isofrequency contours in Figure 2. As depicted in Figure 1a, the proposed structure is derived from a meandered version of the single-mesh connected wires [32] that can be seen as a $3 \mathrm{D}$ extension to the meandered crossed-microstrip-line-based 'fishnet' metasurface studied in [19]. To simplify the parameterization of the unit cell, the mean path of a meandered wire with diameter $d$ is defined to be a sine shape $S(v)=A \sin (N v \pi / p)$ $(v \equiv x, y, z)$, with $p$ and $N$ being the period and the number of turns within one period. The same definition of geometric parameters also applies to the structures in Figure 1b,c. As revealed by the varied contours with different cutting planes in Figure 2a, the lack of spatial symmetry of this structure leads to a strong anisotropy that hinders its use for $3 \mathrm{D}$ lens designs. To enhance symmetry in all spatial directions, another set of wires is added to connect and pair with the original one in each axis, thus a twin-wire mesh is constructed, as illustrated in Figure 1b. Although an improvement of isotropy is evidenced in Figure 2b, the wave propagation inside this structure undergoes severe dispersion due to the presence of cut-off frequency that yields the hyperbolic contour lines. To overcome this problem, an additional copy of the twin-wire mesh is introduced and transposed by half the period in all three Cartesian directions, as shown in Figure 1c. Since the two meshes are disconnected, there is no cut-off frequency, leading to low dispersion and isotropy over a wide bandwidth, as indicated by the spherical contours and their equidistant progression in Figure 2c. Similar phenomena are also reported in [33,34], where these disconnected wire meshes are framed into the so-called 'non-Maxwellian' media. As an alternative viewpoint, one may find an analogy to higher symmetries [38-41], and in particular to glide [37] and 1D twist symmetries [42,43]. When glide symmetries are adequately applied to metasurfaces, they increase the effective refractive index of the unit cells, reduce their dispersion, and increase their isotropy [35,37]. These distinctive properties make 2D glide- 
symmetric structures a suitable candidate for lens applications [20,23,44,45], motivating us to further explore the proposed double-mesh wire medium for 3D meta-lenses.

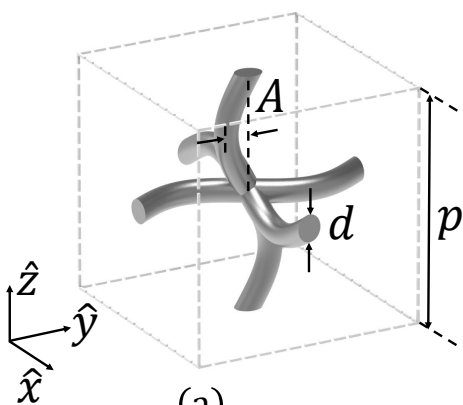

(a)

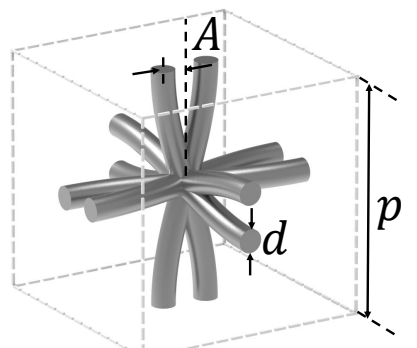

(b)

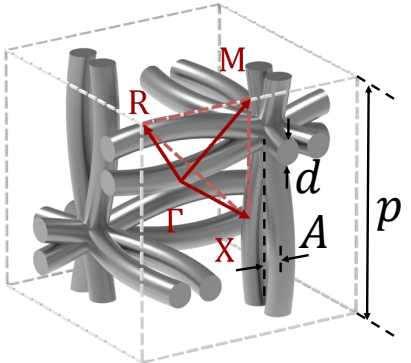

(c)

Figure 1. Evolution of double-mesh twin-wire unit cell: (a) single-wire unit cell; (b) twin-wire unit cell; (c) double-mesh twin-wire unit cell. The mean path of the meandered wire is described by $S(v)=A \sin (N v \pi / p)(v \equiv x, y, z) . p$ is the period of the unit cell and $N$ stands for the number of turns within one period ( $N=1$ in all present schematics).

(a)

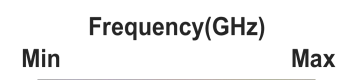

(b)
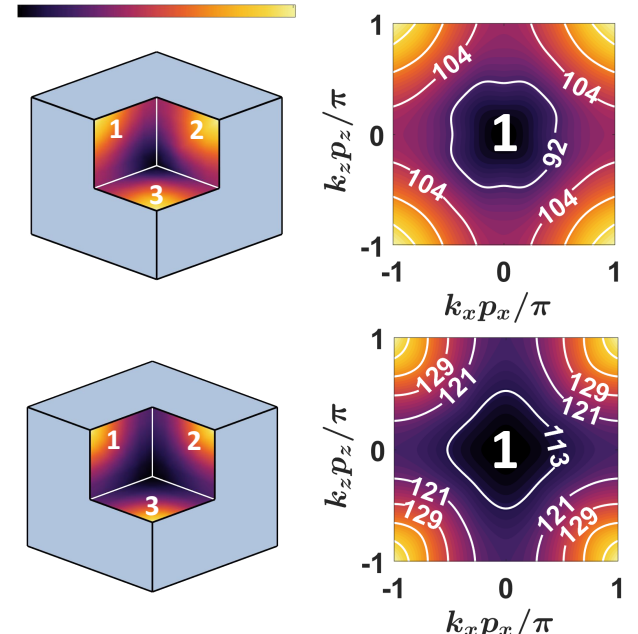

(c)

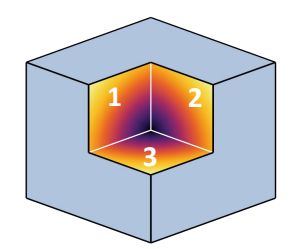

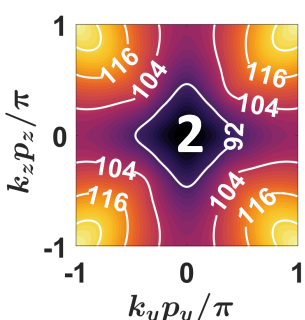
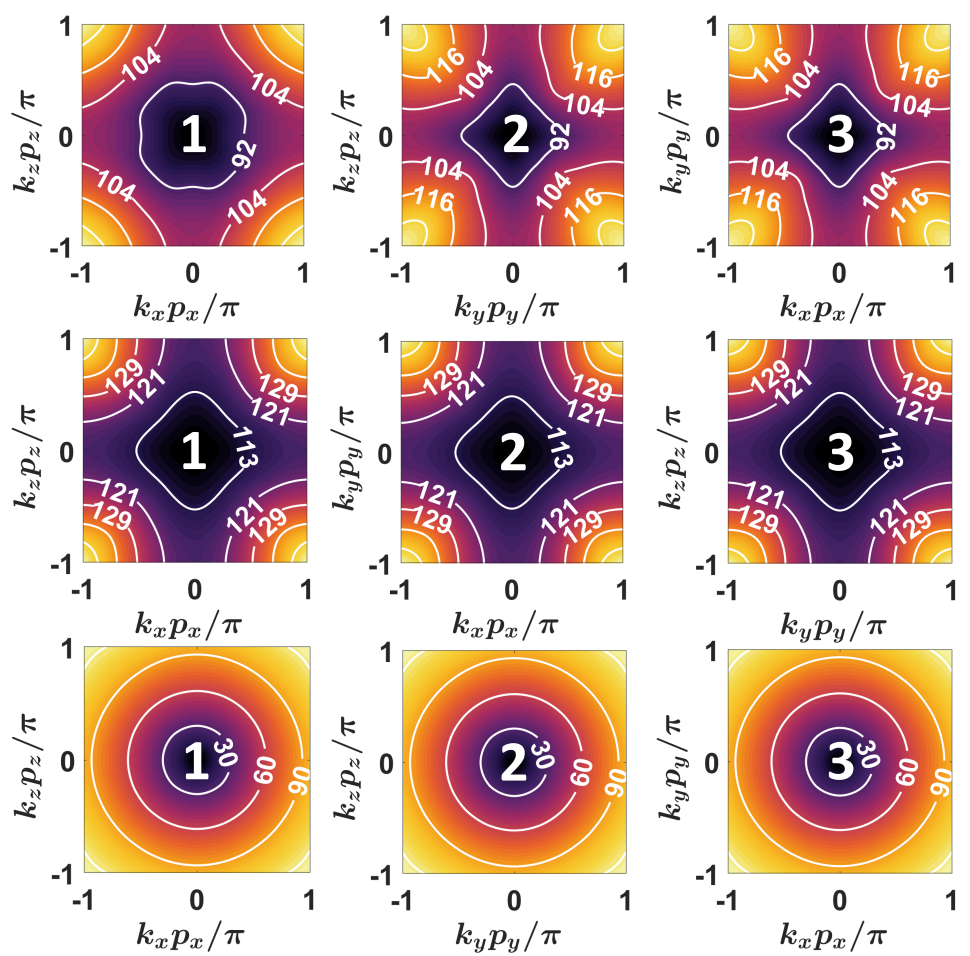

Figure 2. Dispersion analyses of: (a) single-wire unit cell; (b) twin-wire unit cell; (c) double-mesh twin-wire unit cell. The dimensions, as denoted in Figure 1 , are $A=0.1 \mathrm{~mm}, N=1, p=1 \mathrm{~mm}$, and $d=0.1 \mathrm{~mm}$.

A graded-index lens tailors the wave propagation by spatially varying the (effective) refractive index inside the lens medium that provides a sufficiently large tuning range for the index. In a meta-lens, this is usually achieved by locally modifying the geometrical details of the unit cell comprising the medium. In Figure3, we present a parametric study of the effective refractive index of the proposed unit cell. We investigate the impact of the three main geometrical dimensions $A, d, p$. 


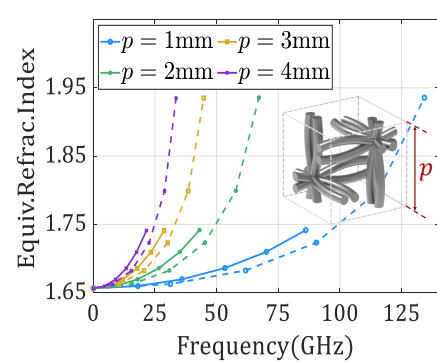

(a)

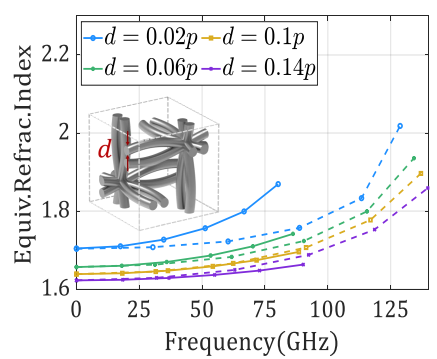

(b)

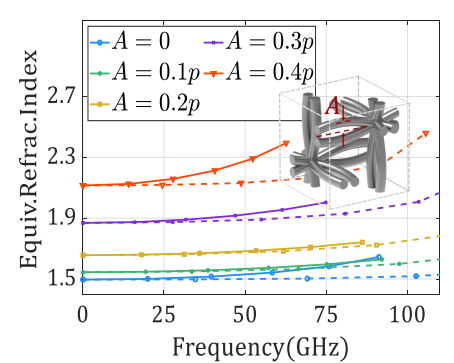

(c)

Figure 3. Effective refractive index of the double-mesh twin-wire unit cell as a function of: (a) period $p$; (b) diameter of wire $d$; (c) amplitude of undulation $A$. The solid lines represent the data for $\overline{\Gamma X}$, and the dashed lines for $\overline{\Gamma R}$. Unless otherwise specified, the dimensions are $A=0.2 \mathrm{~mm}, N=1$, $p=1 \mathrm{~mm}$, and $d=0.06 \mathrm{~mm}$.

In Figure 3a, the effective refractive index for different periods $p$ of the double-wire mesh structure is presented. The period is found to determine the upper limit of operating frequency, beyond which the effective refractive index starts to be dispersive. This frequency dependency is attributed to the fact that the structure can no longer be homogenized into an effective medium for its larger periodicity in comparison to the wavelength at higher frequencies. It is worth noting that the period is not easily varied throughout a graded index lens. In Figure 3b, the effective refractive index for different wire diameters $d$ of the double-wire mesh structure is presented. Changing the wire diameter changes the capacitance of the structure. However, the wire diameter offers only moderate control of the refractive index and cannot be used to design lenses that require a large refractive index range.

The effective refractive index for different amplitudes $A$ of the double-wire mesh structure is presented in Figure 3c. $A$ refers to the amplitude of the undulations of the metallic wires. Therefore, when $A=0$, the wires are straight. The effective refractive index elevates with larger values of $A$ owing to the increased phase delay induced by the sinusoidal meander that enforces the wave to transmit along a longer path, or, in other words, this length increases the inductance. Therefore, when $A=0$, the value of the effective refractive index is the smallest, thus it is regarded as the design reference. As shown in Figure $3 c$, changing $A$ alone from $0.1 p$ to $0.4 p$ gives an index range of around 1.4 , readily sufficient for implementing a Luneburg lens profile $n(r)=n_{0} \sqrt{2-(r / R)^{2}}(R$ being the lens radius and $n_{0}$ the refractive index of the background material, same as that at the periphery of the lens) [46], as discussed in Section 3. Following the same rationale, this range can be further extended for other profiles such as Maxwell fisheye lenses by introducing additional meander turns within the unit cell, i.e., increasing $N$.

\section{Design of Luneburg Lens}

Now, we design a 3D Luneburg lens operating up to $30 \mathrm{GHz}$ based on the proposed unit cell with tailored sinusoidal amplitudes. According to the parametric studies in Figure $3 \mathrm{a}$, the period is chosen to be $p=1 \mathrm{~mm}$ for a good homogenization below $30 \mathrm{GHz}$. The lens radius $R$ is set to be $R=20.5 \mathrm{~mm}$, containing 41 unit cells in its diameter. This configuration produces a fine Cartesian discretization of the ideal index map, as depicted in Figure 4a. To implement this discretized map, the sinusoidal amplitude of the wires in the unit cell is gradually reduced with an increasing distance $r$ from the lens center, and it becomes zero, i.e., the wires are straight, in the lens outer surface. Since the curves of refractive indices in Figure $3 \mathrm{c}$ are almost non-dispersive, the dimensional synthesis is not subject to a strong frequency dependency. Here, it is performed at $24 \mathrm{GHz}$ based on the effective refractive index (normalized to the minimum value of $n=1.4646$ ) as a function of the amplitude $A$, as provided in Figure $4 \mathrm{~b}$. In Figure 4c, we synthesize a dimensional map of the sinusoidal amplitude required at each spatial location in the lens. The full structure of the designed lens is visualized in Figure 5. 


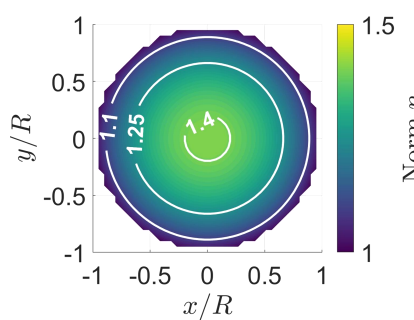

(a)

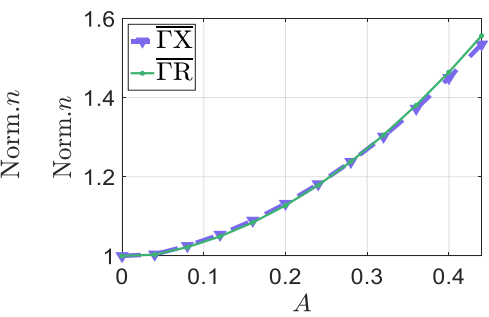

(b)

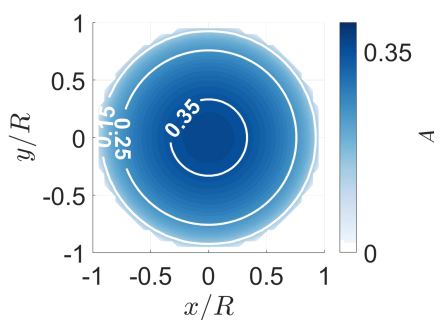

(c)

Figure 4. (a) Discretized refractive index of Lens. (b) Normalized effective refractive index as a function of $A$ at $24 \mathrm{GHz}$. (c) Synthesized dimensional map of the meta-lens.

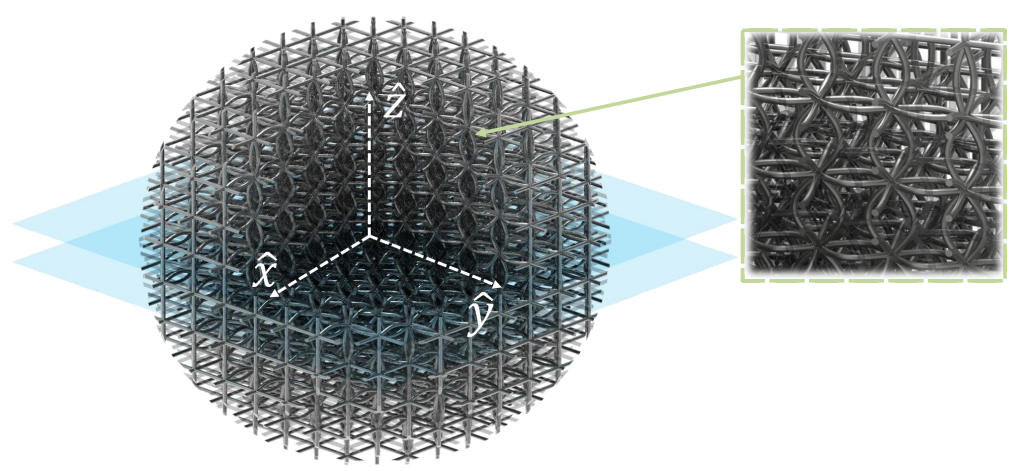

(a)

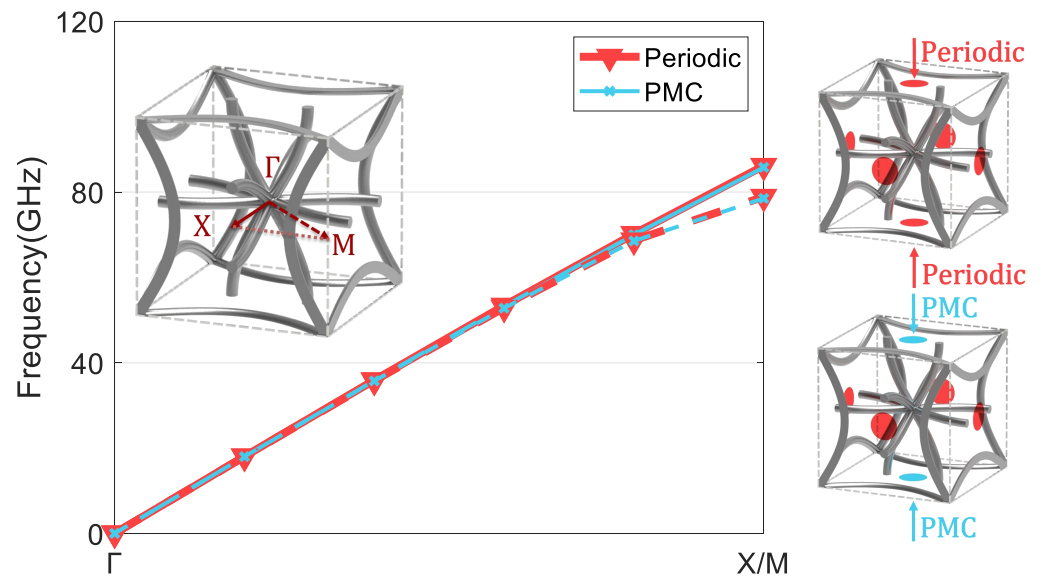

(b)

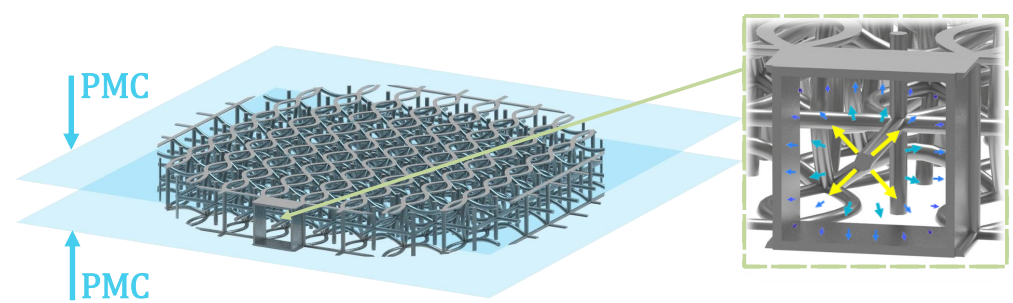

(c)

Figure 5. (a) 3D synthesized meta-Luneburg lens (with zoom-in view of some unit cells). (b) Dispersion diagram of unit cell with different boundary conditions; the dimensions as denoted in Figure 1a are $p=1 \mathrm{~mm}, A=0.2 p$, and $d=0.06 p$, while the solid lines represent the data for $\overline{\Gamma X}$ and the dashed lines for $\overline{\Gamma M}$. The unit cell is defined to be the one in Figure 1c off-shifted by half the period in the $x / y / z$-directions. (c) Equivalent 2D synthesized meta-Luneburg lens (with zoom-in view of port and its field distribution). 
The design of the meta-lens is validated by full-wave simulations in CST Microwave Studio. We note that the propagation characteristics in the ГXM-plane is very similar if the periodic boundary conditions along $\hat{z}$ are replaced with perfect magnetic conductor (PMC) boundaries, as demonstrated in Figure $5 \mathrm{~b}$. Therefore, it suffices to analyze one slab of the lens with PMC boundaries on the top and bottom, as illustrated in Figure 5c. Additionally, the modal field in the twin-wire structure at the boundary of the lattice is TEM-like, similar to the field in a coaxial transmission line. This allows us to excite the lens with a simple feeding structure such as the coaxial port, as illustrated by the inset of Figure $5 \mathrm{c}$.

Note that, since the mode supported by the lens is confined between the metallic wires, it is inherently mismatched from the one in free space. For ease of demonstration, we embed the lens in an effectively homogeneous background composed by the straight wires with $A=0$, i.e., the same as those in the lens exterior. In this way, we obtain the absolute value of electric field $|E|$ contours in the cutting plane $z=0$ as in Figure 6 . Here, the lens produces a uniform wavefront and aperture illumination across a wide operating bandwidth from DC to $30 \mathrm{GHz}$, although only $12-24 \mathrm{GHz}$ are explicitly exemplified for better visualization. This confirms the good isotropy and low dispersion of the proposed double-mesh wire structure studied in Section 2.

The results in Figure 6 are obtained with absorbing boundary conditions around the wire structure. Furthermore, the lens is surrounded by unit cells of the same dimensions as the one on the edge of the lens. This is done to avoid reflections at the border of the lens. In order for the Luneburg lens to be used as an antenna, a structure that matches the mode of the wire mesh to the one in free space must be added around the lens. The design of the matching structure is a future work and is out of the scope of this paper.

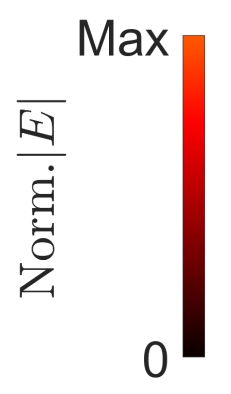

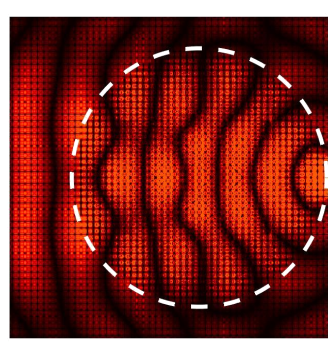

(a)

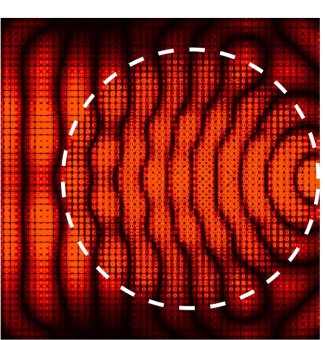

(b)

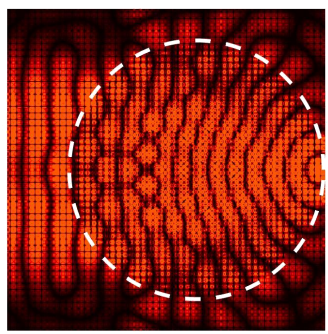

(c)

Figure 6. Full-wave simulated absolute value of $E$-field in the meta-lens whose background is filled with the same unit cell as in the lens exterior at: (a) $12 \mathrm{GHz}$; (b) $18 \mathrm{GHz}$; (c) $24 \mathrm{GHz}$. The lens circumference is outlined by a dashed line.

\section{Conclusions}

We propose a 3D double-mesh twin-wire metamaterial for meta-lenses by introducing additional symmetries in the conventional wire medium. The proposed structure exhibits excellent isotropy and low dispersion over a wide bandwidth without a cut-off frequency. In addition, it provides a wide range of the effective refractive index designable by geometry. These distinctive features are well corroborated by a Luneburg lens that is engineered based on such structure.

Author Contributions: Conceptualization, O.Q.-T.; formal analysis, H.W. and Q.C.; writing-original draft preparation, H.W., Q.C. and O.Z.; writing—review and editing, Q.C., O.Z. and O.Q.-T.; visualization, H.W.; supervision, Q.C., O.Z. and O.Q.-T. All authors have read and agreed to the published version of the manuscript.

Funding: This research received no external funding.

Data Availability Statement: The data that support the findings of this study are available from the corresponding author, Q.C., upon reasonable request.

Acknowledgments: The authors would like to thank Francisco Mesa for helpful discussions. 
Conflicts of Interest: The authors declare no conflict of interest.

\section{References}

1. Schoenlinner, B.; Wu, X.; Ebling, J.; Eleftheriades, G.; Rebeiz, G. Wide-scan spherical-lens antennas for automotive radars. IEEE Trans. Microw. Theory Tech. 2002, 50, 2166-2175. [CrossRef]

2. Wang, Y.; Li, J.; Huang, L.; Jing, Y.; Georgakopoulos, A.; Demestichas, P. 5G Mobile: Spectrum Broadening to Higher-Frequency Bands to Support High Data Rates. IEEE Veh. Technol. Mag. 2014, 9, 39-46. [CrossRef]

3. Sipus, Z.; Komljenovic, T. Multi-shell Radially Symmetrical Lens Antennas. In Aperture Antennas for Millimeter and Sub-Millimeter Wave Applications; Boriskin, A.; Sauleau, R., Eds.; Springer International Publishing: Cham, Switzerland, 2018 ; pp. $37-73$.

4. Quevedo-Teruel, O.; Ebrahimpouri, M.; Ghasemifard, F. Lens Antennas for 5G Communications Systems. IEEE Commun. Mag. 2018, 56, 36-41. [CrossRef]

5. Quevedo-Teruel, O.; Liao, Q.; Chen, Q.; Castillo-Tapia, P.; Mesa, F.; Zhao, K.; Fonseca, N.J. Geodesic lens antennas for 5G and beyond. IEEE Commun. Mag. 2021, in press.

6. Hua, C.; Wu, X.; Yang, N.; Wu, W. Millimeter-wave homogenous cylindrical lens antenna for multiple fan-beam scanning. J. Electromagn. Waves Appl. 2012, 26, 1922-1929. [CrossRef]

7. Peeler, G.; Coleman, H. Microwave stepped-index luneberg lenses. IRE Trans. Antennas Propag. 1958, 6, 202-207. [CrossRef]

8. Thornton, J. Wide-scanning multi-layer hemisphere lens antenna for Ka band. IEE Proc. Microwaves Antennas Propag. 2006, 153, 573-578. [CrossRef]

9. $\quad$ Liang, M.; Ng, W.R.; Chang, K.; Gbele, K.; Gehm, M.E.; Xin, H. A 3-D Luneburg Lens Antenna Fabricated by Polymer Jetting Rapid Prototyping. IEEE Trans. Antennas Propag. 2014, 62, 1799-1807. [CrossRef]

10. Budhu, J.; Rahmat-Samii, Y.; Hodges, R.E.; Hofmann, D.C.; Ruffatto, D.F.; Carpenter, K.C. Three-Dimensionally Printed, Shaped, Engineered Material Inhomogeneous Lens Antennas for Next-Generation Spaceborne Weather Radar Systems. IEEE Antennas Wirel. Propag. Lett. 2018, 17, 2080-2084. [CrossRef]

11. Zhao, Y.Y.; Zhang, Y.L.; Zheng, M.L.; Dong, X.Z.; Duan, X.M.; Zhao, Z.S. Three-dimensional Luneburg lens at optical frequencies. Laser Photonics Rev. 2016, 10, 665-672. [CrossRef]

12. Bjorkqvist, O.; Zetterstrom, O.; Quevedo-Teruel, O. Additive manufactured dielectric Gutman lens. Electron. Lett. 2019, 55, 1318-1320. [CrossRef]

13. Wang, C.; Wu, J.; Guo, Y.X. A 3-D-Printed Multibeam Dual Circularly Polarized Luneburg Lens Antenna Based on QuasiIcosahedron Models for Ka-Band Wireless Applications. IEEE Trans. Antennas Propag. 2020, 68, 5807-5815. [CrossRef]

14. Lou, Y.H.; Zhu, Y.X.; Fan, G.F.; Lei, W.; Lu, W.Z.; Wang, X.C. Design of Ku-Band Flat Luneburg Lens Using Ceramic 3-D Printing. IEEE Antennas Wirel. Propag. Lett. 2021, 20, 234-238. [CrossRef]

15. Ansari, M.; Jones, B.; Zhu, H.; Shariati, N.; Guo, Y.J. A Highly Efficient Spherical Luneburg Lens for Low Microwave Frequencies Realized with a Metal-based Artificial Medium. IEEE Trans. Antennas Propag. 2020, 69, 3758-3770. [CrossRef]

16. Xue, L.; Fusco, V.F. 24 GHz automotive radar planar Luneburg lens. IET Microwaves Antennas Propag. 2007, 1, 624-628. [CrossRef]

17. Liu, P.; Zhu, X.; Zhang, Y.; Jiang, Z.H.; Wang, X.; Hong, W.; Le, T.H. A Novel E-plane-Focused Cylindrical Luneburg Lens Loaded With Metal Grids for Sidelobe Level Reduction. IEEE Trans. Antennas Propag. 2020, 68, 736-744. [CrossRef]

18. Xue, L.; Fusco, V.F. Printed holey plate Luneburg lens. Microw. Opt. Technol. Lett. 2008, 50, 378-380. [CrossRef]

19. Pfeiffer, C.; Grbic, A. A Printed, Broadband Luneburg Lens Antenna. IEEE Trans. Antennas Propag. 2010, 58, 3055-3059. [CrossRef]

20. Quevedo-Teruel, O.; Miao, J.; Mattsson, M.; Algaba-Brazalez, A.; Johansson, M.; Manholm, L. Glide-Symmetric Fully Metallic Luneburg Lens for 5G Communications at Ka-Band. IEEE Antennas Wirel. Propag. Lett. 2018, 17, 1588-1592 [CrossRef]

21. Bantavis, P.; Gonzalez, C.G.; Sauleau, R.; Goussetis, G.; Tubau, S.; Legay, H. Broadband graded index Gutman lens with a wide field of view utilizing artificial dielectrics: A design methodology. Opt. Express 2020, 28, 14648-14661. [CrossRef]

22. Fan, F.; Cai, M.; Zhang, J.; Yan, Z.; Wu, J. Wideband Low-Profile Luneburg Lens Based on a Glide-Symmetric Metasurface. IEEE Access 2020, 8, 85698-85705. [CrossRef]

23. Zetterstrom, O.; Hamarneh, R.; Quevedo-Teruel, O. Experimental Validation of a Metasurface Luneburg Lens Antenna Implemented With Glide-Symmetric Substrate-Integrated Holes. IEEE Antennas Wirel. Propag. Lett. 2021, 20, 698-702. [CrossRef]

24. Kunz, K. Propagation of microwaves between a parallel pair of doubly curved conducting surfaces. J. Appl. Phys. 1954, 25, 642-653. [CrossRef]

25. Liao, Q.; Fonseca, N.; Quevedo-Teruel, O. Compact multibeam fully metallic geodesic Luneburg lens antenna based on non-Euclidean transformation optics. IEEE Trans. Antennas Propag. 2018, 66, 7383-7388. [CrossRef]

26. Fonseca, N.J.G.; Liao, Q.; Quevedo-Teruel, O. Equivalent Planar Lens Ray-Tracing Model to Design Modulated Geodesic Lenses using Non-Euclidian Transformation Optics. IEEE Trans. Antennas Propag. 2020, 68, 3410-3422. [CrossRef]

27. Seol, S.K.; Kim, D.; Lee, S.; Kim, J.H.; Chang, W.S.; Kim, J.T. Electrodeposition-based 3D Printing of Metallic Microarchitectures with Controlled Internal Structures. Small 2015, 11, 3896-3902. [CrossRef]

28. Das, S.; Bourell, D.L.; Babu, S.S. Metallic materials for 3D printing. MRS Bull. 2016, 41, 729-741. [CrossRef]

29. Chieh, J.C.S.; Dick, B.; Loui, S.; Rockway, J.D. Development of a Ku-Band Corrugated Conical Horn Using 3-D Print Technology. IEEE Antennas Wirel. Propag. Lett. 2014, 13, 201-204. [CrossRef]

30. Rebollo, A.; Vaquero, A.F.; Arrebola, M.; Pino, M.R. 3D-Printed Dual-Reflector Antenna With Self-Supported Dielectric Subreflector. IEEE Access 2020, 8, 209091-209100. [CrossRef] 
31. Dimitriadis, A.I.; Debogović, T.; Favre, M.; Billod, M.; Barloggio, L.; Ansermet, J.P.; de Rijk, E. Polymer-Based Additive Manufacturing of High-Performance Waveguide and Antenna Components. Proc. IEEE 2017, 105, 668-676. [CrossRef]

32. Silveirinha, M.; Fernandes, C. Homogenization of 3-D-connected and nonconnected wire metamaterials. IEEE Trans. Microw. Theory Tech. 2005, 53, 1418-1430. [CrossRef]

33. Shin, J.; Shen, J.T.; Fan, S. Three-dimensional electromagnetic metamaterials that homogenize to uniform non-Maxwellian media. Phys. Rev. B 2007, 76, 113101. [CrossRef]

34. Chen, W.J.; Hou, B.; Zhang, Z.Q.; Pendry, J.B.; Chan, C.T. Metamaterials with index ellipsoids at arbitrary k-points. Nat. Commun. 2018, 9, 1-10. [CrossRef]

35. Quevedo-Teruel, O.; Ebrahimpouri, M.; Kehn, M.N.M. Ultrawideband Metasurface Lenses Based on Off-Shifted Opposite Layers. IEEE Antennas Wirel. Propag. Lett. 2016, 15, 484-487. [CrossRef]

36. Arnberg, P.; Barreira Petersson, O.; Zetterstrom, O.; Ghasemifard, F.; Quevedo-Teruel, O. High Refractive Index Electromagnetic Devices in Printed Technology Based on Glide-Symmetric Periodic Structures. Appl. Sci. 2020, 10, 3216. [CrossRef]

37. Quevedo-Teruel, O.; Chen, Q.; Mesa, F.; Fonseca, N.J.; Valerio, G. On the Benefits of Glide Symmetries for Microwave Devices. IEEE J. Microwaves 2021, 1, 457-469. [CrossRef]

38. Crepeau, P.J.; McIsaac, P.R. Consequences of symmetry in periodic structures. Proc. IEEE 1964, 52, 33-43. [CrossRef]

39. Mittra, R.; Laxpati, S. Propagation in a wave guide with Glide refection symmetry. Can. J. Phys. 1965, 43, 353-372. [CrossRef]

40. Kieburtz, R.; Impagliazzo, J. Multimode propagation on radiating traveling-wave structures with glide-symmetric excitation. IEEE Trans. Antennas Propag. 1970, 18, 3-7. [CrossRef]

41. Hessel, A.; Chen, M.H.; Li, R.C.M.; Oliner, A.A. Propagation in periodically loaded waveguides with higher symmetries. Proc. IEEE 1973, 61, 183-195. [CrossRef]

42. Dahlberg, O.; Ghasemifard, F.; Valerio, G.; Quevedo-Teruel, O. Propagation characteristics of periodic structures possessing twist and polar glide symmetries. Eur. Phys. J. Appl. Metamater. 2019. [CrossRef]

43. Castillo-Tapia, P.; Van Gassen, K.; Chen, Q.; Mesa, F.; Sipus, Z.; Quevedo-Teruel, O. Dispersion Analysis of Twist-Symmetric Dielectric Waveguides. Photonics 2021, 8, 206. [CrossRef]

44. Chen, Q.; Giusti, F.; Valerio, G.; Mesa, F.; Quevedo-Teruel, O. Anisotropic glide-symmetric substrate-integrated-holey metasurface for a compressed ultrawideband Luneburg lens. Appl. Phys. Lett. 2021, 118, 084102. [CrossRef]

45. Poyanco, J.M.; Zetterstrom, O.; Castillo-Tapia, P.; Fonseca, N.J.G.; Pizarro, F.; Quevedo-Teruel, O. Two-dimensional glidesymmetric dielectric structures for planar graded-index lens antennas. IEEE Antennas Wirel. Propag. Lett. 2021. [CrossRef]

46. Luneburg, R. Mathematical Theory of Optics; Brown University Press: Providence, RI, USA, 1944; pp. 208-213. 